\title{
Investigating Acetaminophen Hepatotoxicity in Multi-Cellular Organotypic Liver Models
}

\author{
Sophia M. Orbach ${ }^{a}$, Margaret E. Cassin ${ }^{a}$, Marion F. Ehrich ${ }^{\text {b, }}$, \\ Padmavathy Rajagopalan ${ }^{\mathrm{a}, \mathrm{c}, \mathrm{d},{ }^{\star}}$
}
${ }^{2}$ Department of Chemical Engineering, Virginia Tech, Suite 245 Goodwin Hall, 635
Prices Fork Road, Blacksburg, VA, 24061, USA
${ }^{b}$ Department of Biomedical Sciences and Pathobiology, Virginia-Maryland College of Veterinary Medicine, 205 Duck Pond Drive, Blacksburg, VA, 24060, USA
'ICTAS Center for Systems Biology of Engineered Tissue, Virginia Tech, 333 Kelly Hall, 325 Stanger Street, Blacksburg, VA, 24061, USA
${ }^{d}$ School of Biomedical Engineering and Sciences, Virginia Tech, 325 Kelly Hall, 325
Stanger Street, Blacksburg, VA

\section{Author Information}

S. Orbach - smorbach@vt.edu

M. Cassin - mec033@vt.edu

\section{${ }^{*}$ Corresponding authors}

1) M. F. Ehrich

205 Duck Pond Drive, Virginia Tech, Blacksburg, VA 24061

Email: marion@vt.edu, Tel: 1-540-231-4938, FAX: 1-540-231-6333

2) P. Rajagopalan

333 Kelly Hall, Virginia Tech, Blacksburg, VA 24061

Email: padmar@vt.edu, Tel: 1-540-231-4851, FAX: 1-540-231-5022 


\begin{abstract}
In vivo studies clearly demonstrate the participation and subsequent death of nonparenchymal liver cells (NPCs) with corresponding hepatocyte effects. This results in a critical need to investigate how major liver cell types function cohesively during hepatotoxicity. However, virtually no studies replicate these phenomena in vitro. We report the design of multi-cellular three-dimensional (3D) organotypic liver models of primary rat hepatocytes, liver sinusoidal endothelial cells (LSECs) and Kupffer cells (KCs). LSECs and KCs were separated from hepatocytes by a detachable membrane that emulates the physical and chemical properties of the Space of Disse. Acetaminophen (APAP)-induced changes to cellular function and phenotype were investigated. LSECs exhibited approximately $40 \%$ cell death at $20 \mathrm{mM}$ APAP. KCs exhibited decreased interleukin-10 and increased tumor necrosis factor-alpha and interferon-gamma secretion. The secretion of these proteins altered hepatocyte function and signaling. Both LSECs and KCs maintained phenotypic markers. At 20 mM APAP, the 3D models exhibited aspartate aminotransferase to alanine aminotransferase ratios from 2.1-2.5 and $45 \%$ glutathione depletion, corresponding to what is seen in vivo. At 10 and $20 \mathrm{mM}$ APAP, the 3D models exhibited cell death, primarily through necrosis. Therefore, the $3 \mathrm{D}$ cultures described in this report demonstrate significant potential as realistic models for hepatotoxicity studies.
\end{abstract}

\title{
KEYWORDS
}

Acetaminophen; liver; organotypic culture models; multi-cellular

\section{ABBREVIATIONS}


$2 \mathrm{DHL}=2 \mathrm{D}$ co-culture of hepatocytes and liver sinusoidal endothelial cells, $3 \mathrm{DHL}=3 \mathrm{D}$ culture of hepatocytes, collagen/hyaluronic acid multilayer and liver sinusoidal endothelial cells, $3 D H L K=3 D$ culture of hepatocytes, collagen/hyaluronic acid multilayer, liver sinusoidal endothelial cells and Kupffer cells, ALT = alanine aminotransferase, $\mathrm{APAP}=$ acetaminophen, $\mathrm{AST}=$ aspartate aminotransferase, $\mathrm{CS}=$ collagen sandwich, $\mathrm{CYP}=$ cytochrome P450, DMEM = Dulbecco's modified Eagle medium, $\mathrm{ECM}=$ extracellular matrix, $\mathrm{GSH}=$ glutathione, $\mathrm{HM}=$ hepatocyte monolayer, $\mathrm{HRP}=$ horseradish peroxidase, $\mathrm{HSC}$ = hepatic stellate cells, IFN- $y=$ interferon gamma, $\mathrm{IL}-10=$ interleukin $10, \mathrm{KCs}=$ Kupffer cells, LSECs $=$ liver sinusoidal endothelial cells, MTT $=$ thiazolyl blue tetrazolium bromide, NAPQI $=N$-acetyl- $p$-benzoquinoneimine NPCs $=$ non-parenchymal liver cells, PBS $=$ phosphate buffered saline, PVDF = polyvinylidene fluoride, TNF- $\alpha=$ tumor necrosis factor alpha 


\section{INTRODUCTION}

The liver is primarily responsible for the biotransformation of many toxicants and xenobiotics (Arias et al., 2001). Such biotransformation can induce hepatotoxicity, a phenomenon investigated in vivo and in vitro (Godoy et al., 2013; Kaplowitz, 2005; Klassen, 2013). The metabolism of drugs and chemicals is accomplished through continuous interactions between parenchymal (hepatocytes) and non-parenchymal liver cells (NPCs). The NPCs are primarily liver sinusoidal endothelial cells (LSECs), Kupffer cells (KCs) and hepatic stellate cells (HSCs) (Arias et al., 2001). Hepatocytes are separated from LSECs and KCs by a protein-rich interface called the Space of Disse. This interface is composed of extracellular matrix (ECM) components and plays a critical role in the transfer of nutrients and exchange of signaling molecules between the different cell types.

In vivo toxicity evaluations have clearly illustrated protective and inflammatory roles of NPCs when the liver is exposed to toxicants (Godoy et al., 2013; LeCluyse et al., 2012). LSECs serve as the first line of defense when a toxicant enters the hepatic microcirculation (Ito et al., 2003; Sorensen et al., 2015). These cells die upon exposure to toxicants, thereby protecting hepatocytes. Alterations to NPC functions and phenotypes occur upon liver injury in a process known as activation (Bataller and Brenner, 2005; Godoy et al., 2013; Ito et al., 2003). For example, during liver injury, KCs secrete pro- and anti-inflammatory cytokines that recruit neutrophils to the site of injury and regulate oxidative stress and inflammation (Godoy et al., 2013; Ito et al., 2003; Ju et al., 2002). However, the mechanisms by which NPCs simultaneously ameliorate and exacerbate hepatotoxicity are not understood. 
Despite the important contributions of NPCs towards hepatotoxicity, in vitro studies have focused on monocultures, such as the hepatocyte monolayer (HM) or collagen sandwich (CS) (Godoy et al., 2013; LeCluyse et al., 2012). These models do not capture the wide range of heterotypic inter-cellular interactions in the liver. Two-dimensional (2D) cocultures have primarily focused on the effects of fibroblasts, epithelial cells, endothelial cells, or NPCs on hepatocyte function and phenotype (Bhatia et al., 1999; Godoy et al., 2013; LeCluyse et al., 2012). Some 2D co-culture studies have reported hepatocyteNPC interactions in the presence of toxicants (DeLeve et al., 1997; Milosevic et al., 1999; Nelson et al., 2015). These 2D systems are easy to assemble but are not representative of the native three-dimensional (3D) microenvironment.

To date, 3D models have been utilized to investigate the maintenance of hepatic phenotypes and hepatocyte toxicity (Godoy et al., 2013). Common 3D liver models include bioreactors (Allen et al., 2005; Mueller et al., 2013), microfluidic devices (Toh et al., 2009; Trietsch et al., 2013), gel entrapped hepatocytes (Lan et al., 2010; Shen et al., 2007), spheroids (Gunness et al., 2013; Messner et al., 2013), and scaffolds (Kostadinova et al., 2013; Toyoda et al., 2012). To the best of our knowledge, these models have not been used to elucidate specific changes to NPCs upon toxicant exposure. In one study, Kostadinova et al. (2013) assembled a 3D hepatic model for toxicity with a NPC fraction (seeded on a Nylon scaffold) above hepatocytes. The NPCs proliferated and secreted ECM proteins forming a Space of Disse mimic. However, detailed investigations into NPC-specific death and phenotypic changes were not reported. In summary, toxicity investigations using 3D liver models have not demonstrated how specific NPCs respond to a toxicant and how they alter signaling mechanisms. These gaps in knowledge can be addressed by the design of in vitro models that mimic the hepatic 3D microenvironment, where each cell type can be easily 
isolated for further analyses. Such models may enable accelerated investigations on efficacy and toxicity for drugs and chemicals.

We previously reported the design and assembly of 3D organotypic hepatic models comprised of primary rat hepatocytes, LSECs and KCs (Kim et al., 2010; Kim and Rajagopalan, 2010; Larkin et al., 2013). These liver-mimetic models contained a detachable membrane that mimicked the physical and chemical properties of the Space of Disse. These models maintained hepatic functions and phenotypes over 16 days. A unique feature of these 3D models was that the ratio of hepatocytes to NPCs was identical to in vivo, demonstrating similarity to the native hepatic microenvironment (Larkin et al., 2013). Additionally, cytochrome P450 (CYP) 1A1/2 and CYP3A enzyme activities were maintained (Kim and Rajagopalan, 2010; Larkin et al., 2013). Since CYP expression is critical for biotransformation, such 3D models are promising for conducting toxicity evaluations.

We report a detailed toxicity investigation on 3D organotypic hepatic models comprised of primary rat hepatocytes (H), LSECs (L), KCs (K), and a multilayer composed of alternating layers of type I collagen and hyaluronic acid. The 3D liver models of hepatocytes and LSECs (3DHL) or hepatocytes, LSECs and KCs (3DHLK) were compared to 2D co-cultures (2DHL) and hepatocyte monocultures (HM and CS). We tested the liver models by administering a prototypic hepatotoxicant, acetaminophen (APAP). Although APAP is nontoxic at therapeutic doses, acute drug induced liver injury occurs upon overdose (McGill and Jaeschke, 2013). In vivo, APAP is oxidized by CYP2E1 to the active metabolite $N$-acetyl-p-benzoquinoneimine (NAPQI). Excess NAPQI results in the depletion of glutathione $(\mathrm{GSH})$, generation of reactive oxygen species and eventually necrosis (Hinson et al., 2010; McGill and Jaeschke, 2013). 2D 
and 3D models were investigated to determine the extent and mechanisms of cell death, changes in GSH and the ratio of aspartate aminotransferase (AST) to alanine aminotransferase $(A L T)$. Cytokine secretion was investigated to determine if APAP toxicity resulted in inflammation in these models. A unique feature of these 3D models is the ability to monitor changes in NPC counts and phenotypes in response to the toxicant thereby providing a better understanding of APAP hepatotoxicity. Our goal was to determine how closely the $3 \mathrm{D}$ models compared to the biotransformation of APAP reported in rodents.

\section{MATERIALS AND METHODS}

Dulbecco's modified Eagle medium (DMEM), phosphate buffered saline (PBS, 10X), penicillin-streptomycin, human plasma fibronectin, primary KCs, $10 \%$ tris-glycine gels, and polyvinylidine fluoride (PVDF) membranes were purchased from Thermo Fisher Scientific (Carlsbad, CA). 4-(2-hydroxyethyl)-piperazine-1-ethanesulfonic acid, glucagon, calcium chloride, hydrocortisone, sodium dodecyl sulfate, hydrogen peroxide, glutaraldehyde, calf thymus DNA, hyaluronic acid, sulfuric acid, collagenase type IV, protease inhibitor cocktail, and thiazolyl blue tetrazolium bromide (MTT) were obtained from Sigma-Aldrich (St. Louis, MO). All other chemicals were ordered from Fisher Scientific (Pittsburgh, PA) unless otherwise stated. All experimental procedures were approved by and conducted in accordance with Virginia Tech's Institutional Biosafety Committee.

\section{Extraction of Type I Collagen}

Collagen was extracted from rat tails using previously described procedures (Ford et al., 2015). Briefly, tendons were dissolved in acetic acid and centrifuged at 13,000 $\times$ g. A 
$30 \%(\mathrm{w} / \mathrm{v})$ sodium chloride solution was slowly added to the supernatant and centrifuged at $8,500 \times g$. The resulting solution was dialyzed in $1 \mathrm{mN}$ hydrochloric acid. The final collagen suspension was sterilized with chloroform and maintained at a pH of 3.1.

\section{Isolation and Culture of Hepatocytes and LSECs}

Primary hepatocytes and LSECs were isolated from female Lewis rats (175-199 g; Harlan Laboratories, Indianapolis, IN) using a two-step in situ collagenase perfusion method (Kim et al., 2010; Kim and Rajagopalan, 2010; Larkin et al., 2013). Animal care and the procedure for liver excision from rats were approved by and conducted in accordance with the Virginia Tech Institutional Animal Care and Use Committee. A typical surgical and isolation procedure resulted in 100-150 million hepatocytes with viability $\geq 97 \%$ as determined through trypan blue exclusion. LSEC separation was conducted by differential adhesion and the cells were cultured in fibronectin-coated flasks (Kim and Rajagopalan, 2010; Larkin et al., 2013).

Hepatocytes were seeded as monolayers at $0.5 \times 10^{6}$ cells/well in a 12-well plate on collagen gels (Larkin et al., 2013). Wells were coated with $0.25 \mathrm{ml}$ collagen and gelation was induced by increasing the temperature to $37^{\circ} \mathrm{C}$. In CS models, a second layer of collagen was added $24 \mathrm{~h}$ post-hepatocyte seeding.

All cultures were maintained in hepatocyte medium consisting of DMEM supplemented with $10 \%(\mathrm{v} / \mathrm{v})$ heat-inactivated fetal bovine serum, $100 \mathrm{U} / \mathrm{ml}$ penicillin-streptomycin, 0.5 $\mathrm{IU} / \mathrm{ml}$ insulin (MP Biomedicals, Solon, $\mathrm{OH}$ ), $14.3 \mathrm{ng} / \mathrm{ml}$ glucagon, and $7.6 \mu \mathrm{g} / \mathrm{ml}$ hydrocortisone. Spent culture medium was collected at the end of each $24 \mathrm{~h}$ period and maintained at $-80{ }^{\circ} \mathrm{C}$ until analyzed. All cultures were maintained in a humidified gas environment at $37^{\circ} \mathrm{C}$ containing $10 \%$ carbon dioxide. 


\begin{abstract}
Assembly of Multi-cellular Models
Primary rat hepatocytes were seeded on collagen gels. Collagen/hyaluronic acid multilayers were placed directly above the hepatocytes and hydrated with hepatocyte medium for approximately $1 \mathrm{~h}$ (refer to Supplementary Data for assembly of the multilayers). The assembly of these models occurred $72 \mathrm{~h}$ post hepatocyte seeding to allow the formation of a confluent monolayer. LSECs and KCs were seeded and cultured on hydrated multilayers. Initial seeding ratios were 1:20 (LSECs:hepatocytes) and 1:10 (KCs:hepatocytes). For 2D co-cultures, LSECs were seeded directly on the hepatocytes at a ratio of 1:20 (LSECs:hepatocytes) $72 \mathrm{~h}$ post-hepatocyte seeding (Larkin et al., 2013).
\end{abstract}

When referring to a specific multi-cellular culture the following notation is used. The absence or presence of the collagen/hyaluronic acid multilayer is denoted by a "2D" or "3D" prefix, respectively. Rat hepatocytes, LSECs and KCs are abbreviated as "H", "L" and "K", respectively. A visual description of the types of multi-cellular models is presented in Figure 1. For a detailed description of changes in hepatocyte and NPC counts in each culture, refer to the Supplementary Figure S1.

\title{
Administering APAP to Hepatic Cultures
}

APAP (CAS Number: 103-90-2) was dissolved in hepatocyte medium and administered on day 4 of culture for $24 \mathrm{~h}$. The drug was administered at concentrations of $0 \mathrm{mM}$ (untreated; control), $10 \mathrm{mM}, 20 \mathrm{mM}$, and $40 \mathrm{mM}$. These concentrations were derived from in vivo rodent pharmacokinetic properties (Bachmann et al., 1996; Belanger et al., 1987; Lee and Blaufox, 1985). Additionally, these concentrations are consistent with 
those used for in vivo and in vitro studies (Table 1). Spent culture medium and cell lysates were extracted after the 24 h APAP incubation.

\section{Determining the Mechanism of Hepatocyte Death in Response to APAP}

The numbers of live, necrotic and apoptotic cells were determined using a commercially available kit (Apoptotic, Necrotic, and Healthy Cells Quantification Kit; Biotium, Hayward, CA). $24 \mathrm{~h}$ after APAP treatment, cells were incubated with $50 \mu \mathrm{L} / \mathrm{ml}$ of Hoechst 33342 (cell nuclei), ethidium homodimer III (necrotic) and FITC-annexin V (apoptotic). Imaging was performed on a Nikon TE2000 inverted microscope.

\section{Measuring Overall Cell Viability}

The overall cell viability of control and APAP-treated hepatic cultures was assayed using the MTT assay (Mosmann, 1983). Cultures were incubated for $4 \mathrm{~h}$ with $0.5 \mathrm{mg} / \mathrm{ml}$ MTT in phenol-red free DMEM. The resulting formazan crystals formed in live cells were dissolved in $0.04 \mathrm{~N}$ hydrochloric acid (diluted in isopropanol). The absorbance was measured at $570 \mathrm{~nm}$.

\section{Changes in Hepatic Cell Counts Upon APAP Administration}

To obtain information on changes in the number of LSECs and KCs in the multi-cellular models, the cells were labeled with nontoxic, fluorescent, membrane permeable dyes prior to seeding. LSECs and KCs were incubated with red (LSECs; PKH 26 Red Fluorescence Cell Linker Kit; Sigma-Aldrich) and green (KCs; PKH 2 Green Fluorescence Cell Linker Kit; Sigma-Aldrich) dye, respectively. Cytoplasmic labeling was performed as per the manufacturer's protocols and imaged using a Nikon TE2000 inverted microscope. LSEC and KC viability in monocultures was determined by trypan blue exclusion. Hepatocyte viability in multi-cellular models was determined by 
measuring protein content.

\section{Imaging LSECs and KCs by Immunostaining}

2DHL, 3DHL and 3DHLK cultures were fixed in 2\% (w/v) glutaraldehyde in PBS (1X) and sequentially exposed to $0.1 \%(\mathrm{v} / \mathrm{v})$ Triton $\mathrm{X}-100$ and a $1 \%(\mathrm{w} / \mathrm{v})$ bovine serum albumin in PBS blocking solution with rabbit serum (Kim and Rajagopalan, 2010; Larkin et al., 2013). The cultures were then incubated with a mouse anti-rat sinusoidal endothelial-1 antibody (CD32b; ImmunoBiological Laboratories, Minneapolis, MN) to identify LSECs. Thereafter, either a FITC-conjugated monoclonal ED-2 antibody (CD163; AbD Serotec, Bio-Rad, Hercules, CA) was added to identify KCs or a FITCconjugated monoclonal CD31 antibody (Abcam, Cambridge, MA) was added to identify dedifferentiated LSECs. This was followed by incubation with a TRITC-conjugated secondary rabbit anti-mouse IgG antibody (Abcam) to visualize LSECs. Imaging was conducted on a Zeiss LSM confocal microscope (Oberkochen, Germany).

\section{Measuring the Concentrations of ALT and AST}

The concentrations of ALT and AST in spent hepatocyte culture medium were determined using commercially available kits (ALT (SGPT) Reagent (Colorimetric, Endpoint Method; Teco Diagnostics, Anaheim, CA) and AST (SGOT) Reagent (Colorimetric, Endpoint Method; Teco Diagnostics)). Manufacturer recommended protocols were followed. The absorbance was measured at either $505 \mathrm{~nm}(\mathrm{ALT})$ or 530 nm (AST). A calibrator (Teco Diagnostics) was used to convert absorbance values to enzyme concentrations.

\section{Measuring Changes in GSH in Hepatocytes}


In 2DHL cultures, LSECs were separated from the hepatocytes using a magnetic cell separation process (Larkin et al., 2013). Briefly, the CD32b antibody was conjugated to Dynabeads $^{\circledR}$ (CELLection ${ }^{\mathrm{TM}}$ Kit; Invitrogen Thermo Fisher Scientific) (Tokairin et al., 2002). The LSEC fraction adhered to the beads and was separated using a magnet (DynaMag $^{\mathrm{TM}}$, Invitrogen Thermo Fisher Scientific). The supernatant contained the hepatocyte fraction. In the 3DHL and 3DHLK cultures, the hepatocyte fraction was obtained upon removal of the multilayer.

Hepatocytes were lysed in PBS (1X) containing $1 \mathrm{mM}$ ethylene diamine tetra-acetic acid maintained at $4{ }^{\circ} \mathrm{C}$. The lysates were centrifuged at $10,000 \times g$ for $15 \mathrm{~min}$, and the supernatant was collected and stored at $-20{ }^{\circ} \mathrm{C}$. Total GSH was measured using a commercially available colorimetric kinetic assay kit (Glutathione Assay Kit; Cayman Chemicals, Ann Arbor, Ml). The absorbance was measured at $405 \mathrm{~nm}$ every 5 min for $30 \mathrm{~min}$. The total GSH concentration was determined by calibrating to a standard curve.

\section{Western Immunoblotting for CYP2E1}

The hepatocyte fraction from each culture was lysed and incubated with a protease inhibitor cocktail of 4-(2-aminoethyl) benzenesulfonyl fluoride hydrochloride, aprotinin, bestatin, E-64, leupeptin, and pepstain A. The total protein concentration was measured using a commercially available kit (Coomassie (Bradford) Protein Assay Kit; Thermo Scientific). Gel electrophoresis was performed with a 10\% tris-glycine gel followed by protein transfer to a PVDF membrane. CYP2E1 and $\beta$-actin (housekeeping protein) were identified through exposure to primary antibodies for CYP2E1 (rabbit polyclonal anticytochrome P450 enzyme CYP2E1; EMD Millipore, Billerica, MA) and $\beta$-actin (rabbit monoclonal anti- $\beta$-actin; Cell Signaling Technology, Danvers, MA), respectively. The membrane was exposed to a secondary horseradish peroxidase (HRP)-conjugated 
antibody (anti-rabbit IgG; Bio-Rad). The membrane was developed with a chemiluminescent HRP substrate (Clarity Western ECL Substrate; Bio-Rad) and imaged on a Chemidoc ${ }^{\text {TM }}$ XRS+ system (Bio-Rad). Image analysis was conducted using Image Lab $^{\mathrm{TM}}$ software (Bio-Rad).

\begin{abstract}
Assaying the Levels of Pro- and Anti-Inflammatory Cytokines
Interleukin 10 (IL-10) concentration was measured using a commercially available kit (Rat IL-10 ELISA Kit; Thermo Scientific). To measure the levels of tumor necrosis factor alpha (TNF- $\alpha$ ), spent culture medium samples were concentrated through the use of Vivaspin $^{\mathrm{TM}} 2$ Centrifugal Concentrators (Sartorius, Goettingen, Germany). The concentration of TNF- $\alpha$ was measured using a commercially available kit (Rat TNFalpha Quantikine ELISA Kit; R\&D Systems, Minneapolis, MN). The interferon gamma (IFN- $\mathrm{y}$ ) concentration was measured using a commercially available kit (IFN- $\mathrm{y}$ ELISA Kit, Rat; Thermo Scientific). The concentrations of IL-10, TNF- $\alpha$ and IFN- $\gamma$ were individually determined by calibrating to standard curves.
\end{abstract}

\title{
Statistical Analysis
}

Statistical significance and $p$-values were calculated by a Student's t-test, assuming unequal variance while applying the Bonferroni (multiple hypothesis testing) correction. Statistical significance, unless specifically noted, compared untreated (0 mM APAP) to each treatment condition (10,20 and 40 mM APAP). Reported $p$-values are the products of individual $p$-values and the number of comparisons. For all significance testing $\alpha=$ 0.05. All data are reported as mean \pm standard deviation; $n$ denotes sample size (number of separate experiments). The data presented in this report were collected and analyzed from up to five independent experiments, with three replicates per experiment. 


\section{RESULTS}

Most in vitro studies report changes in viability, hepatocyte function and CYP expression (Allen et al., 2005; Khetani and Bhatia, 2008; Toh et al., 2009). While this information is very helpful, the role of NPCs has largely been ignored in in vitro hepatotoxicity investigations. We report how APAP affects i) the model as a whole, ii) each cell type and iii) the secretion of signaling molecules. The incorporation of NPCs can begin to address the gap between in vivo studies and in vitro models. We have broadly classified our results with hepatocyte monocultures (HM and CS) and 2D and 3D multi-cellular liver models (2DHL, 3DHL, and 3DHLK) into three sections that provide information at different levels of investigation:
A. Changes in viability of each cell type
B. Maintenance of NPC phenotypes
C. Changes in liver toxicity markers

\section{Changes in Viability of Each Cell Type in Response to APAP}

Changes in overall viabilities of each culture model upon APAP administration were investigated using the MTT assay. HM, CS and 2DHL cultures only exhibited significant cell death at $40 \mathrm{mM}$ APAP (approximately 30\%; $p<0.05$ ) (Figure 2). In contrast, the 3DHL and 3DHLK models exhibited approximately $10 \%$ cell death at $10 \mathrm{mM}$ and approximately $20 \%$ cell death at $20 \mathrm{mM}(p<0.05)$. Viability decreased by approximately $45 \%$ in the 3D models at $40 \mathrm{mM}$ APAP $(p<0.05)$. These results demonstrate that the 3D models exhibited greater sensitivity to APAP. 
Since the MTT assay measures the viability of the entire culture, detailed studies were undertaken to determine effects of APAP exposure on each cell type. APAP exposure of hepatocytes decreased protein content in the 3D models by $20 \%$ whereas hepatocyte protein levels in the 2DHL cultures decreased by $32 \%$ (Table 2). The effects of APAP on LSECs and KCs were greater than on hepatocytes.

Apoptosis/necrosis staining was conducted to investigate the mechanism of hepatocyte death (Figure 3). In the CS and 3D models, the primary mode of cell death was necrosis, consistent with prior studies on APAP (Hinson et al., 2010; McGill and Jaeschke, 2013). At 20 mM APAP, apoptotic cells in CS (3.2 $\pm 0.8 \%)$, 3DHL (7.4 \pm $1.0 \%)$ and 3DHLK cultures $(11.5 \pm 3.2 \%)$ were a small fraction of total cell death. In contrast, HM and 2DHL models exhibited a high percentage of apoptotic cells. Even untreated cultures exhibited $23.3 \pm 6.6 \%(\mathrm{HM})$ and $15.0 \pm 4.8 \%(\mathbf{D H L})$ apoptotic cells.

At all concentrations of APAP, LSEC and KC monolayers exhibited at most $10 \%$ cell death (Table 2). Hepatocytes in monocultures easily change phenotype, so the absence of APAP sensitivity in monolayers may be attributed to their de-differentiation, inability to metabolize APAP and the lack of exposure to subsequent NAPQI-related effects (Hinson et al., 2010; McGill and Jaeschke, 2013; Sellaro et al., 2007; Zinchenko et al., 2006). In the multi-cellular liver models, LSEC death was significantly higher in the 3D cultures compared to the 2DHL cultures in response to APAP and exceeded that of hepatocytes (Table 2). This is consistent with in vivo studies that reported significant LSEC death upon APAP administration (DeLeve et al., 1997; Ito et al., 2003). LSEC death at 10 and $20 \mathrm{mM}$ APAP was $33 \%$ and $40 \%$ in the 3DHLK cultures, respectively, and $14 \%$ and $18 \%$ in the 2DHL model, respectively. In the 3DHLK cultures, KCs appeared to proliferate 
upon exposure to 10 and $20 \mathrm{mM}$ APAP (approximately $15 \%$ and $34 \%$, respectively) relative to untreated samples $(p<0.05)$. Similar proliferation of KCs has been observed upon liver injury (Antoniades et al., 2012; Zigmond et al., 2014).

\section{Maintenance of NPC Phenotypes in Response to APAP}

As no previous studies have reported APAP-induced changes to NPC phenotypes, phenotypic markers of NPCs were investigated for multi-cellular models using immunofluorescence staining for CD32b (LSECs) and CD163 (KCs). LSEC counts were measured using CD32b staining (phenotypic marker) and a non-specific cytoplasmic dye. The difference in number of LSECs measured with the two dyes was statistically insignificant in the 3D models (Supplementary Table S1). In contrast, untreated 2DHL cultures exhibited fewer CD32b-expressing cells compared to 3DHL or 3DHLK models (Figure 4). The number of CD32b-expressing LSECs was statistically lower in the 2DHL models than those counted using cytoplasmic staining (Supplementary Table S1), suggesting the possibility of dedifferentiation. In the 3DHLK cultures, KCs stained for CD163 after exposure to all APAP concentrations, with some decrease observed upon 40 mM APAP, a similar feature was exhibited when the cytoplasmic stain was used. The similarity of staining characteristics of NPCs in the 3D models suggests that they maintain phenotypic markers (Supplementary Table S1).

We further investigated the decrease in CD32b-expressing cells associated with an increase in LSECs expressing CD31 (a marker for dedifferentiated LSECs) (Figure 5; further information in Supplementary Table S2) (Elvevold et al., 2008). Cells exhibiting both CD31 and CD32b were identified as dedifferentiated. A similar observation was made with the $2 \mathrm{D}$ co-cultures, as they exhibited the largest number of dedifferentiated LSECs. CD31-expressing cells in 2DHL cultures were up to 7-fold higher than 
corresponding 3DHLK models. Similarly, CD31-expressing cells in 3DHL cultures were up to 2.6-fold greater than in 3DHLK models. Based on these comparisons, it was evident that KCs improved the maintenance of the LSEC phenotype.

\section{Changes in Liver Toxicity Markers in Response to APAP Investigating Changes in ALT and AST Concentrations}

The concentrations of ALT and AST in spent culture medium were measured to assess hepatic injury. In serum, an AST/ALT ratio greater than 2.0 is a clinical marker for liver damage (Dufour et al., 2000). This ratio ranged from $0.64-1.22$ in the $2 \mathrm{D}$ models upon exposure to 10 and 20 mM APAP (Figure 6a). In the 3DHL models, the AST/ALT ratio was $1.33 \pm 0.25$ and $2.12 \pm 0.08$ at 10 and $20 \mathrm{mM} \mathrm{APAP,}$ respectively. In the 3DHLK cultures, this ratio was $1.73 \pm 0.46$ and $2.46 \pm 0.17$ at these concentrations. While all models exhibited AST/ALT ratios greater than 2.0 at $40 \mathrm{mM} \mathrm{APAP,} \mathrm{this} \mathrm{ratio} \mathrm{was} 4.28 \pm$ 0.69 and $4.67 \pm 0.48$ in the $3 \mathrm{DHL}$ and 3 DHLK cultures, respectively.

\section{Measuring Changes in Hepatocyte GSH and CYP2E1}

The concentration of total GSH was measured in hepatocyte lysates (Figure 6b). GSH decreased $22-40 \%$ in all cultures upon administration of 10 mM APAP $(p<0.05)$. At 20 mM APAP, GSH decreased by approximately $70 \%(p<0.05)$ and $45 \%(p<0.05)$ in 2D and 3D models, respectively. The presence of NPCs in the 3D models may have contributed to the maintenance of GSH. Western immunoblotting of CYP2E1 was conducted on hepatocyte lysates and was detected in the 3D cultures treated with APAP. HM, CS and 2DHL cultures did not exhibit well-defined bands for CYP2E1 at any APAP concentration (Figure 7a). 


\section{Measuring Changes in Pro- and Anti-inflammatory Cytokines}

IL-10 (anti-inflammatory), TNF- $\alpha$ (pro-inflammatory) and IFN- $\gamma$ (pro-inflammatory) are cytokines secreted primarily by KCs, although there is evidence of secretion by other liver cells (Hinson et al., 2010). Cytokine levels were measured to determine association of APAP toxicity with inflammation. In untreated HM, CS, 2DHL, and 3DHL cultures, the concentrations of these cytokines were statistically insignificant from fresh culture medium (Table 3). In untreated 3DHLK cultures, cytokine concentrations were at least 35-fold (IL-10), 2.5-fold (TNF- $\alpha$ ) and 1.6-fold (IFN- $\mathrm{y}$ ) higher than the other cultures. These data suggest that the three cytokines were primarily secreted by KCs in vitro, which was subsequently verified by determination of cytokine secretion in spent culture medium from 3DHLK cultures. IL-10 decreased approximately 4-fold after exposure to 20 mM APAP (Figure 7b), whereas IFN-y and TNF- $\alpha$ increased by approximately $50 \%$ and $11 \%$, respectively (Figures $7 \mathrm{c}$ and $\mathbf{7 d}$ ). At $40 \mathrm{mM}$ APAP, due to excessive KC death, all cytokine concentrations decreased. Furthermore, AST secretion in the 3DHLK models was approximately 1.5 -fold higher than the $3 \mathrm{DHL}$ cultures. The proposed interaction of these observations is presented in Figure 7e (Ishida et al., 2002), which suggests KC proliferation at this concentration could have resulted in increased proinflammatory cytokine secretion. This increase in IFN- $\gamma$ upon APAP exposure may relate to the increase in hepatocyte apoptosis and higher AST secretion.

To determine if the proximity of hepatocytes and NPCs in the 3DHLK models contributed to changes in cytokine secretion, values obtained from 3DHLK cultures were compared to those from hepatic cell monocultures (Supplementary Table S3). Cytokine levels of fresh medium and hepatocyte or LSEC monocultures were found to be statistically insignificant. IFN- $y$ was significantly higher in the 3DHLK models than KC 
monocultures from 0-20 mM APAP. At 40 mM APAP, there was significantly higher TNF$\alpha$ in the 3DHLK cultures than in KC monocultures.

\section{DISCUSSION}

The gap between in vivo and in vitro toxicity studies can lead to differing results. In this study, responses of a 3D multi-cellular liver model were examined in the presence of a prototypic hepatotoxicant, APAP. We used this model to demonstrate differences in changes to parenchymal and non-parenchymal cells when exposed to this toxicant.

When the liver is exposed to toxicants, NPCs become activated, resulting in altered function and phenotype (Bataller and Brenner, 2005; Godoy et al., 2013; Hinson et al., 2010; Ito et al., 2003). For example, LSECs contract and lose their fenestrae and scavenging abilities (Godoy et al., 2013; Hinson et al., 2010; Ito et al., 2003). Activated KCs proliferate and secrete pro- and anti-inflammatory cytokines (Godoy et al., 2013; Hinson et al., 2010). The varying profiles and altered phenotypes of NPCs during liver toxicity are important factors to consider when testing the efficacy or toxicity of drugs and chemicals.

In order to isolate the effect of NPCs, 3D models were also assembled that contained only a collagen/hyaluronic acid multilayer above the hepatocyte layer (no NPCs; denoted as $3 \mathrm{DH})$. The response to APAP in the $3 \mathrm{DH}$ cultures was similar to $2 \mathrm{D}$ cultures (Supplementary Figure S2). These data suggest that the inclusion of NPCs was critical to the maintenance of sensitivity to APAP that occurred within the $3 \mathrm{D}$ models. 
Closer examination of each cell type in the 3DHL and 3DHLK models revealed additional information. For example, hepatocyte death in these models occurred primarily via necrosis, identical to studies conducted in vivo (Hinson et al., 2010; McGill and Jaeschke, 2013). However, increased secretion of IFN- $\gamma$ by KCs resulted in approximately $11 \%$ apoptosis in the 3DHLK models (Ishida et al., 2002). In contrast, HM and 2DHL cultures exhibited significant apoptosis even in the absence of APAP suggesting that they are less suitable for studies on mechanisms associated with cell death. Although hepatocytes in the 3D models exhibited increased expression of CYP2E1 and other markers of toxicity, the improved albumin secretion suggests that the NPCs acted to support the maintenance of hepatocyte-specific functions. The loss of CYP2E1 protein in the $2 \mathrm{D}$ models is consistent with reports in the literature that demonstrate 2D co-cultures do not maintain hepatocyte CYP2E1 expression (Donato et al., 1994). The lack of expression of this enzyme could explain the insensitivity to APAP in the 2D cultures (Figure 7A; Supplementary Figure S3).

In vivo studies have reported that the sinusoidal endothelium suffers the greatest initial impact upon exposure to hepatotoxicants (DeLeve et al., 1997; Ito et al., 2003; Liu et al., 2013). In the present study, this was only observed in the 3D cultures. Additionally, only the 3D models consistently expressed CD32b, a marker of LSEC phenotype (Geraud et al., 2010). Interestingly, the addition of KCs resulted in the simultaneous maintenance of LSEC phenotype and increased LSEC sensitivity to APAP. Although the exact mechanisms for LSEC death are not fully understood, we hypothesize that the decrease in IL-10 may have played a role. There is evidence that LSECs undergo classical and alternative activation due to exposure to cytokines (Liu et al., 2013). Specifically, removal of IL-10 from culture medium resulted in the transition of LSEC phenotype from M2 (anti-inflammatory) to M1 (pro-inflammatory). Such LSEC activation is also 
associated with the production of inducible nitric oxide synthase, which is directly correlated to cell death in response to APAP (Bourdi et al., 2002). This could explain the increased LSEC death exhibited in the 3DHLK models and merits further investigation.

The proliferation and activation of KCs results in increased inflammation (Antoniades et al., 2012; Zigmond et al., 2014). In the present study, cell death in KC monocultures was minimal and did not correlate to APAP concentration, suggesting loss of sensitivity. In direct contrast, KC proliferation and death in the 3DHLK models was dependent on APAP concentration. Furthermore, APAP treatment resulted in up to a 9-fold decrease in IL-10 with a concomitant increase in TNF- $\alpha$ and IFN- $\gamma$. Taken together, these results indicate that in response to APAP, KC activation results in inflammation in the 3DHLK models.

In vivo hepatotoxicity studies have demonstrated that increased liver toxicity occurs through the production of pro-inflammatory cytokines (Gardner et al., 2003) or decrease of anti-inflammatory cytokines (Bourdi et al., 2002). Changes in cytokine concentrations in the 3DHLK models indicate a shift in KC phenotype from M2 to M1 upon exposure to APAP. Paracrine signaling appears to have modulated cytokine secretion. For example, at $40 \mathrm{mM}$ APAP, the TNF- $\alpha$ secretion was higher in the 3DHLK models than the KC monocultures. This can be attributed to increased TNF- $\alpha$ secretion from hepatocytes in the presence of KCs (Cao et al., 2005; Godoy et al., 2013; Nastevska et al., 1999). The secretion of IFN-y was also significantly higher in the 3DHLK models. These results suggest that the increases in TNF- $\alpha$ and IFN- $\gamma$ were facilitated through heterotypic intercellular interactions and merit further investigation. 
It is critically important to note that at $20 \mathrm{mM}$ APAP ( $800 \mathrm{mg} / \mathrm{kg}$ APAP, Table 1), only the 3D models exhibited AST/ALT ratios from 2.1-2.5, identical to values obtained in vivo (Ahmed et al., 2011; Kikkawa et al., 2006). At 10 and 20 mM APAP, the increased AST/ALT ratio in the 3DHLK models parallels that reported previously (Ishida et al., 2002). In vivo, rats treated with $800 \mathrm{mg} / \mathrm{kg}$ APAP exhibited a $40 \%$ reduction in $\mathrm{GSH}$ (Ahmed et al., 2011). At the corresponding in vitro concentration $(20 \mathrm{mM})$ only the 3D models exhibited a 45\% decrease in GSH. At longer time-points only the 3D models exhibited sensitivity to APAP. This property makes the 3D models relevant for investigations that require longer testing periods (further information can be found in the Supplementary Figure S4).

The strong relationships between the 3D models and data obtained in vivo demonstrate the potential use of these multi-cellular models for drug testing. These appear to be excellent 3D in vitro hepatic models for emulating changes observed in hepatic cell types, closely mimicking events that occur in livers of APAP-treated rodents. The ability to include and isolate NPCs in organotypic liver models described here provides capability to conduct systematic measurements of toxicant effects on each cell type. The future inclusion of HSCs in the 3D models will further increase insights into HSChepatocyte interactions that modulate response to toxicants. Experiments designed to delineate intricate signaling pathways between NPCs and hepatocytes will provide additional insights. Our current experiments demonstrate that the 3D organotypic models offer potential for a new avenue to bridge the gap between in vitro and in vivo toxicity investigations.

\section{ACKNOWLEDGEMENTS}




\begin{abstract}
We gratefully acknowledge financial support from the United States Environmental Protection Agency (US EPA Grant: R834998, P.R. and M.F.E.) and the National Science Foundation (DBI 1062380, P.R.). We also acknowledge financial support from the Institute for Critical Technologies and Applied Sciences at Virginia Tech and the Computational Tissue Engineering Graduate Education Program at Virginia Tech.
\end{abstract}

\title{
AUTHOR DISCLOSURE STATEMENT
}

No competing financial interests exist.

*M. F. Ehrich Email: marion@vt.edu, Tel: 1-540-231-4938, FAX: 1-540-231-6333

*P. Rajagopalan: Email: padmar@vt.edu, Tel: 540-231-4851, Fax: 540-231-5022, 


\section{REFERENCES}

Ahmed, M.M., et al., 2011. Aldo-keto reductase-7A protects liver cells and tissues from acetaminophen-induced oxidative stress and hepatotoxicity. Hepatology 54, 1322-1332.

Allen, J.W., et al., 2005. In vitro zonation and toxicity in a hepatocyte bioreactor. Toxicol Sci 84, 110-119.

Antoniades, C.G., et al., 2012. Source and characterization of hepatic macrophages in acetaminophen-induced acute liver failure in humans. Hepatology 56, 735-746.

Arias, I.M., et al., 2001. The Liver: Biology and Pathology, 4th ed. Lippincott Williams and Wilkins, Philadelphia, PA.

Bachmann, K., et al., 1996. Scaling basic toxicokinetic parameters from rat to man. Environ Health Perspect 104, 400-407.

Bataller, R., Brenner, D.A., 2005. Liver fibrosis. J Clin Invest 115, 209-218.

Belanger, P.M., et al., 1987. Time-dependent variations in the organ extraction ratios of acetaminophen in rat. J Pharmacokinet Biopharm 15, 133-143.

Bhatia, S.N., et al., 1999. Effect of cell-cell interactions in preservation of cellular phenotype: cocultivation of hepatocytes and nonparenchymal cells. FASEB J 13, 18831900.

Bourdi, M., et al., 2002. Protection against acetaminophen-induced liver injury and lethality by interleukin 10: role of inducible nitric oxide synthase. Hepatology 35,289 298.

Cao, Q., et al., 2005. Cytochrome P4502E1 primes macrophages to increase TNF-alpha production in response to lipopolysaccharide. Am J Physiol Gastrointest Liver Physiol 289, G95-107. 
DeLeve, L.D., et al., 1997. Sinusoidal endothelial cells as a target for acetaminophen toxicity. Direct action versus requirement for hepatocyte activation in different mouse strains. Biochem Pharmacol 53, 1339-1345.

Donato, M.T., et al., 1994. Cytochrome P450 activities in pure and co-cultured rat hepatocytes. Effects of model inducers. In Vitro Cell Dev Biol Anim 30A, 825-832.

Dufour, D.R., et al., 2000. Diagnosis and monitoring of hepatic injury. II. Recommendations for use of laboratory tests in screening, diagnosis, and monitoring. Clin Chem 46, 2050-2068.

Elvevold, K., et al., 2008. The liver sinusoidal endothelial cell: a cell type of controversial and confusing identity. Am J Physiol Gastrointest Liver Physiol 294, G391-400.

Ford, A.J., et al., 2015. Designing a fibrotic microenvironment to investigate changes in human liver sinusoidal endothelial cell function. Acta Biomater 24, 220-227.

Gardner, C.R., et al., 2003. Exaggerated hepatotoxicity of acetaminophen in mice lacking tumor necrosis factor receptor-1. Potential role of inflammatory mediators. Toxicol Appl Pharmacol 192, 119-130.

Geraud, C., et al., 2010. Liver sinusoidal endothelium: a microenvironment-dependent differentiation program in rat including the novel junctional protein liver endothelial differentiation-associated protein-1. Hepatology 52, 313-326.

Godoy, P., et al., 2013. Recent advances in 2D and 3D in vitro systems using primary hepatocytes, alternative hepatocyte sources and non-parenchymal liver cells and their use in investigating mechanisms of hepatotoxicity, cell signaling and ADME. Arch Toxicol 87, 1315-1530.

Gunness, P., et al., 2013. 3D organotypic cultures of human HepaRG cells: a tool for in vitro toxicity studies. Toxicol Sci 133, 67-78.

Hinson, J.A., et al., 2010. Mechanisms of acetaminophen-induced liver necrosis. Handb Exp Pharmacol 196, 369-405. 
Ishida, Y., et al., 2002. A pivotal involvement of IFN-gamma in the pathogenesis of acetaminophen-induced acute liver injury. FASEB J 16, 1227-1236.

Ito, Y., et al., 2003. Early hepatic microvascular injury in response to acetaminophen toxicity. Microcirculation 10, 391-400.

Ju, C., et al., 2002. Protective role of Kupffer cells in acetaminophen-induced hepatic injury in mice. Chem Res Toxicol 15, 1504-1513.

Kaplowitz, N., 2005. Idiosyncratic drug hepatotoxicity. Nat Rev Drug Discov 4, 489-499.

Khetani, S.R., Bhatia, S.N., 2008. Microscale culture of human liver cells for drug development. Nat Biotechnol 26, 120-126.

Kikkawa, R., et al., 2006. In vivo hepatotoxicity study of rats in comparison with in vitro hepatotoxicity screening system. J Toxicol Sci 31, 23-34.

Kim, Y., et al., 2010. The design of in vitro liver sinusoid mimics using chitosanhyaluronic acid polyelectrolyte multilayers. Tissue Eng Part A 16, 2731-2741.

Kim, Y., Rajagopalan, P., 2010. 3D hepatic cultures simultaneously maintain primary hepatocyte and liver sinusoidal endothelial cell phenotypes. PLoS One 5, e15456.

Klassen, C.D., 2013. Casarett and Doull's Toxicology: The Basic Science of Poisons. McGraw-Hill, New York, NY.

Kostadinova, R., et al., 2013. A long-term three dimensional liver co-culture system for improved prediction of clinically relevant drug-induced hepatotoxicity. Toxicol Appl Pharmacol 268, 1-16.

Lan, S.F., et al., 2010. Long-term cultivation of HepG2 liver cells encapsulated in alginate hydrogels: a study of cell viability, morphology and drug metabolism. Toxicol In Vitro 24, 1314-1323.

Larkin, A.L., et al., 2013. Designing a multicellular organotypic 3D liver model with a detachable, nanoscale polymeric Space of Disse. Tissue Eng Part C Methods 19, 875884. 
LeCluyse, E.L., et al., 2012. Organotypic liver culture models: meeting current challenges in toxicity testing. Crit Rev Toxicol 42, 501-548.

Lee, H.B., Blaufox, M.D., 1985. Blood volume in the rat. J Nucl Med 26, 72-76.

Liu, L., Klaassen, C.D., 1996. Different mechanism of saturation of acetaminophen sulfate conjugation in mice and rats. Toxicol Appl Pharmacol 139, 128-134.

Liu, Y., et al., 2013. Classical and alternative activation of rat hepatic sinusoidal endothelial cells by inflammatory stimuli. Exp Mol Pathol 94, 160-167.

McGill, M.R., Jaeschke, H., 2013. Metabolism and disposition of acetaminophen: recent advances in relation to hepatotoxicity and diagnosis. Pharm Res 30, 2174-2187.

Messner, S., et al., 2013. Multi-cell type human liver microtissues for hepatotoxicity testing. Arch Toxicol 87, 209-213.

Milosevic, N., et al., 1999. Kupffer cell-mediated differential down-regulation of cytochrome P450 metabolism in rat hepatocytes. Eur J Pharmacol 368, 75-87.

Mosmann, T., 1983. Rapid colorimetric assay for cellular growth and survival: application to proliferation and cytotoxicity assays. J Immunol Methods 65, 55-63.

Mueller, D., et al., 2013. Real-time in situ viability assessment in a 3D bioreactor with liver cells using resazurin assay. Cytotechnology 65, 297-305.

Nastevska, C., et al., 1999. Impairment of TNF-alpha expression and secretion in primary rat liver cell cultures by acetaminophen treatment. Toxicology 133, 85-92.

Nelson, L.J., et al., 2015. Acetaminophen cytotoxicity is ameliorated in a human liver organotypic co-culture model. Sci Rep 5, 17455.

Sellaro, T.L., et al., 2007. Maintenance of hepatic sinusoidal endothelial cell phenotype in vitro using organ-specific extracellular matrix scaffolds. Tissue Eng 13, 2301-2310.

Shen, C., et al., 2007. An in vitro model for long-term hepatotoxicity testing utilizing rat hepatocytes entrapped in micro-hollow fiber reactor. Biochemical Engineering Journal $34,267-272$. 
Sorensen, K.K., et al., 2015. Liver Sinusoidal Endothelial Cells. Compr Physiol 5, 17511774 .

Toh, Y.C., et al., 2009. A microfluidic 3D hepatocyte chip for drug toxicity testing. Lab Chip 9, 2026-2035.

Tokairin, T., et al., 2002. A highly specific isolation of rat sinusoidal endothelial cells by the immunomagnetic bead method using SE-1 monoclonal antibody. J Hepatol 36, 725733.

Toyoda, Y., et al., 2012. Acetaminophen-induced hepatotoxicity in a liver tissue model consisting of primary hepatocytes assembling around an endothelial cell network. Drug Metab Dispos 40, 169-177.

Trietsch, S.J., et al., 2013. Microfluidic titer plate for stratified 3D cell culture. Lab Chip 13, 3548-3554.

Wang, S., et al., 2008. Three-dimensional primary hepatocyte culture in synthetic selfassembling peptide hydrogel. Tissue Eng Part A 14, 227-236.

Zigmond, E., et al., 2014. Infiltrating monocyte-derived macrophages and resident kupffer cells display different ontogeny and functions in acute liver injury. J Immunol 193, 344-353.

Zinchenko, Y.S., et al., 2006. Hepatocyte and kupffer cells co-cultured on micropatterned surfaces to optimize hepatocyte function. Tissue Eng 12, 751-761. 
Table 1: Commonly used doses and concentrations of APAP in rodent models. The corresponding concentrations to each in vivo dose were calculated through the use of pharmacokinetic parameters (Ahmed et al., 2011; Allen et al., 2005; Khetani and Bhatia, 2008; Kikkawa et al., 2006; Messner et al., 2013; Nastevska et al., 1999; Shen et al., 2007; Toh et al., 2009).

\begin{tabular}{|c|c|}
\hline Dose (mg/kg) & Concentration (mM) \\
\hline 300 & $\sim 10$ \\
\hline 800 & $\sim 20$ \\
\hline 1,500 & $\sim 40$ \\
\hline
\end{tabular}


Table 2: Changes in cellular responses to APAP in 2D and 3D multi-cellular liver models and NPC monolayers. Effect of APAP on hepatocytes was determined through changes in protein content. Effects on LSECs and KCs were determined by cell counts, comparing numbers prior and $24 \mathrm{~h}$ post APAP-treatment. Results expressed as percent of control (0 mM APAP), mean $\pm \mathrm{SD}, n=3$.

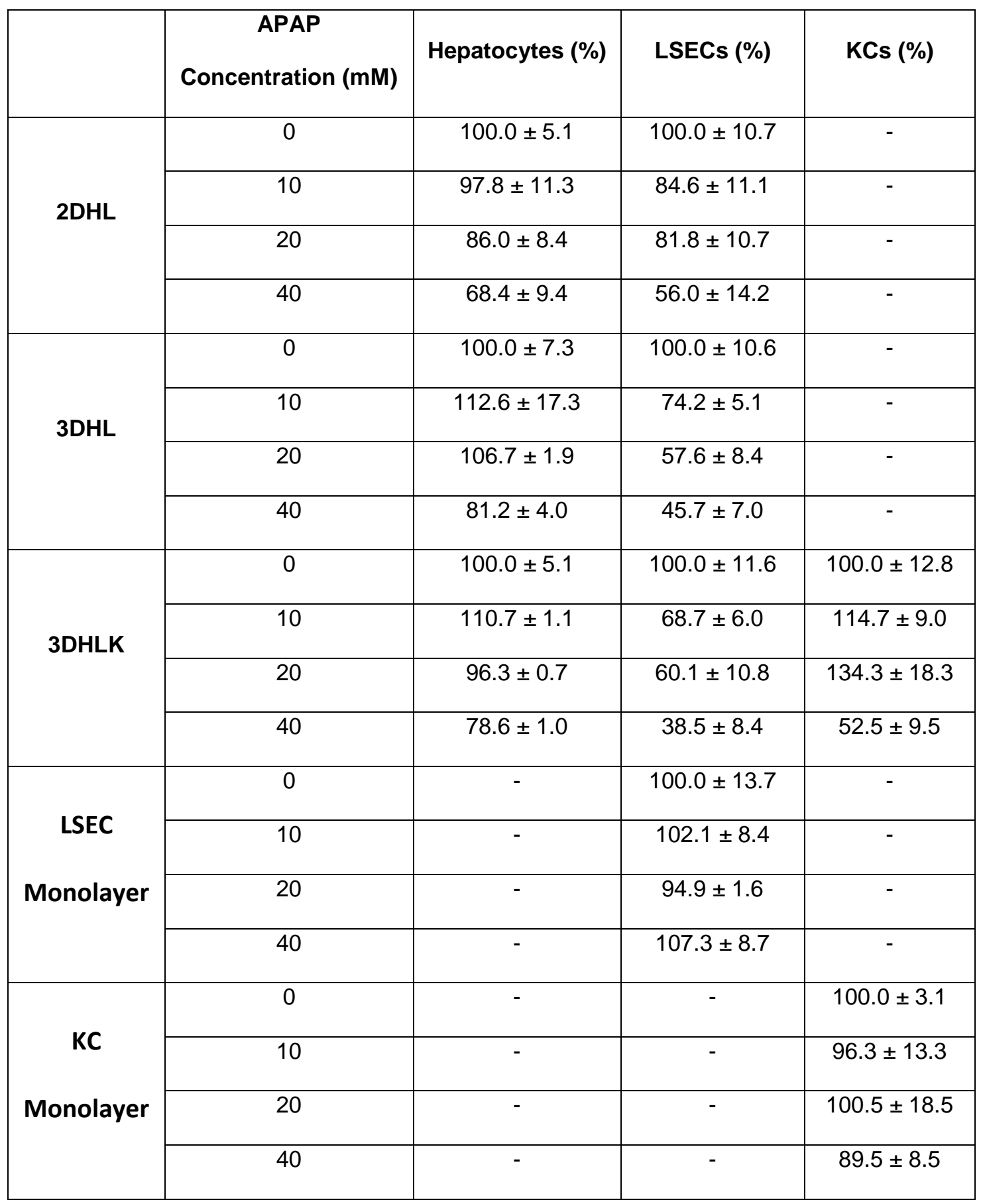


Table 3: Cytokine concentration in unused hepatocyte medium (denoted as "medium") and untreated ( $0 \mathrm{mM}$ APAP) liver models, mean $\pm \mathrm{SD}, n=3$. Cytokine is listed as "undetectable" when the concentration was below the detection limit provided by the manufacturer.

\begin{tabular}{|c|c|c|c|}
\hline Model & IL-10 $(\mathbf{p g} / \mathbf{m l})$ & TNF- $\alpha(\mathbf{p g} / \mathbf{m l})$ & IFN-y $(\mathbf{p g} / \mathbf{m l})$ \\
\hline Medium & $3.06 \pm 0.08$ & Undetectable & $3.38 \pm 0.31$ \\
\hline $\mathrm{HM}$ & $3.34 \pm 0.72$ & Undetectable & $3.90 \pm 0.26$ \\
\hline $\mathrm{CS}$ & $3.42 \pm 1.03$ & Undetectable & $3.42 \pm 0.07$ \\
\hline 2DHL & $3.50 \pm 0.24$ & Undetectable & $3.97 \pm 0.52$ \\
\hline 3DHL & $3.73 \pm 0.67$ & Undetectable & $2.98 \pm 0.15$ \\
\hline 3DHLK & $132.50 \pm 32.32$ & $8.61 \pm 0.86$ & $6.21 \pm 0.39$ \\
\hline
\end{tabular}


Figure 1

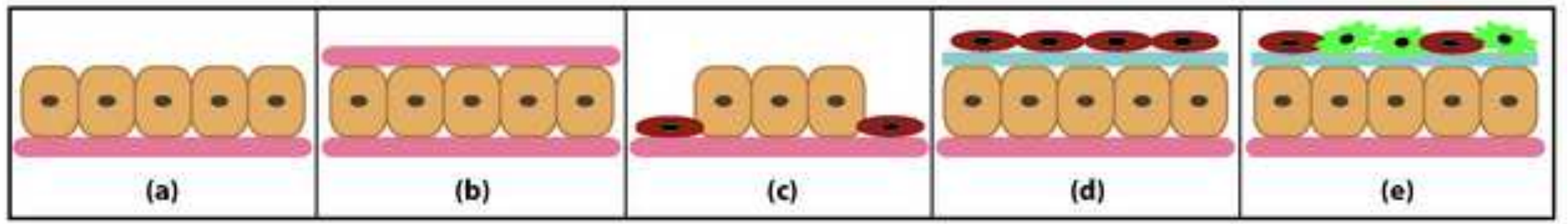

- Hepatocytes $\Leftrightarrow$ LSECS $\because$ KCs Collagen Gel Collagen/Hyaluronic Acid Multilayer

(f)

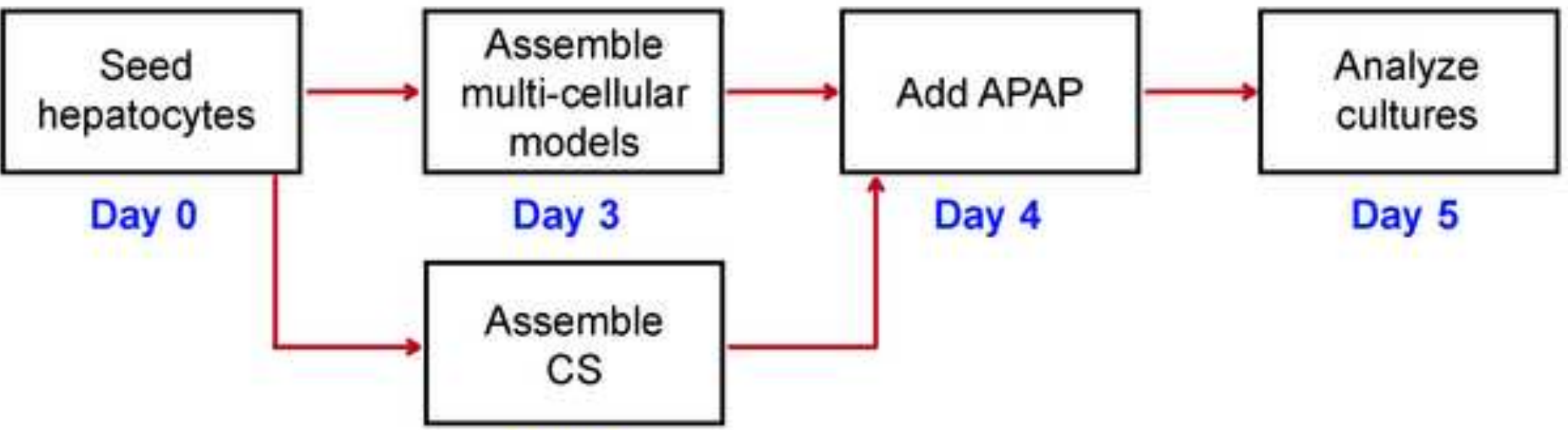

Day 1

\begin{tabular}{lll}
\hline Model & Abbreviation & Schematic \\
\hline Hepatocyte monolayer & HM & Fig 1a \\
Collagen sandwich & CS & Fig 1b \\
2D co-culture of hepatocytes and LSECs & $2 \mathrm{DHL}$ & Fig 1c \\
3D model with hepatocytes, multilayer and LSECs & $3 \mathrm{DHL}$ & Fig 1d \\
3D model with hepatocytes, multilayer, LSECs, and KCs & $3 \mathrm{DHLK}$ & Fig 1e \\
\hline
\end{tabular}


Figure 2

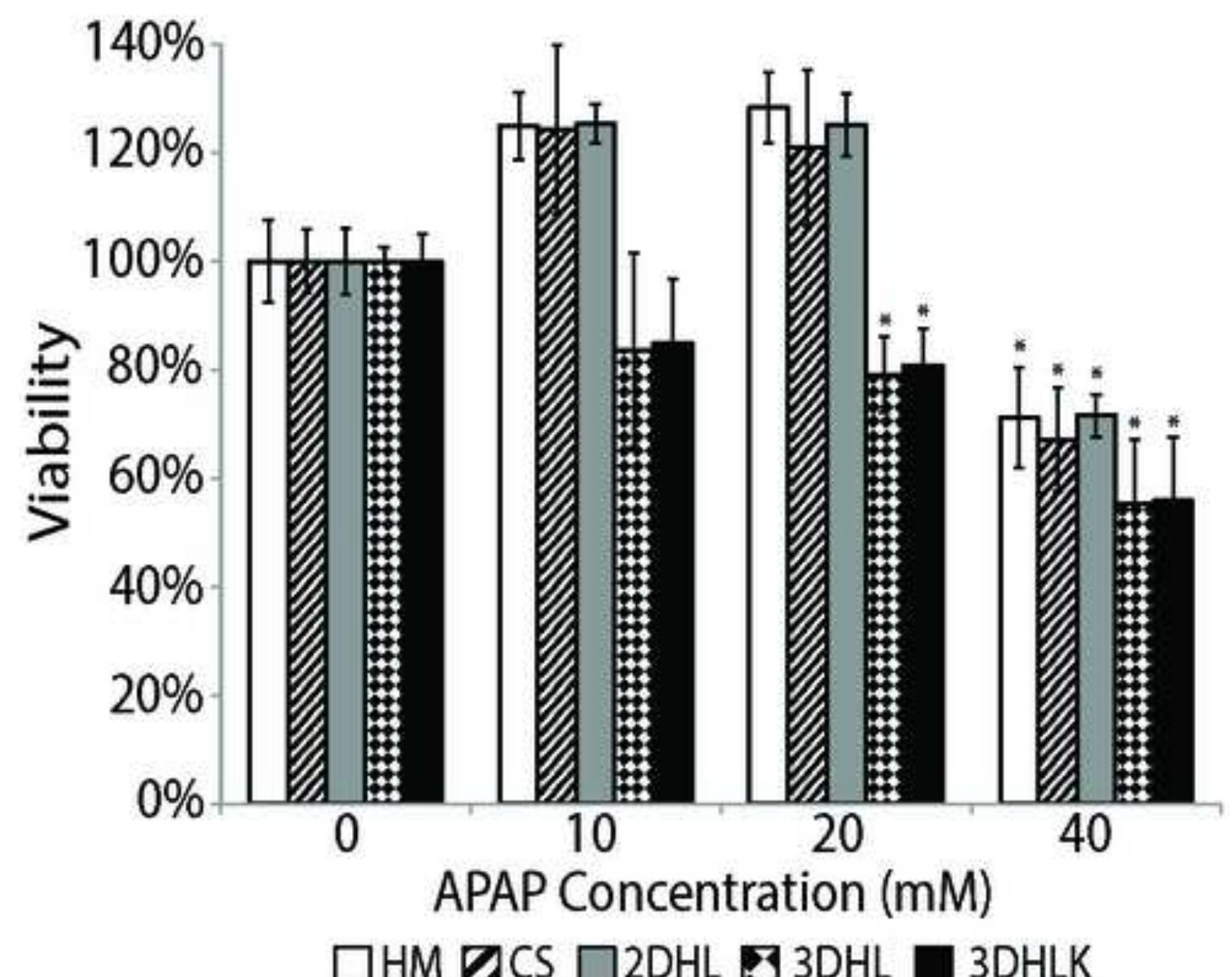


Figure 3

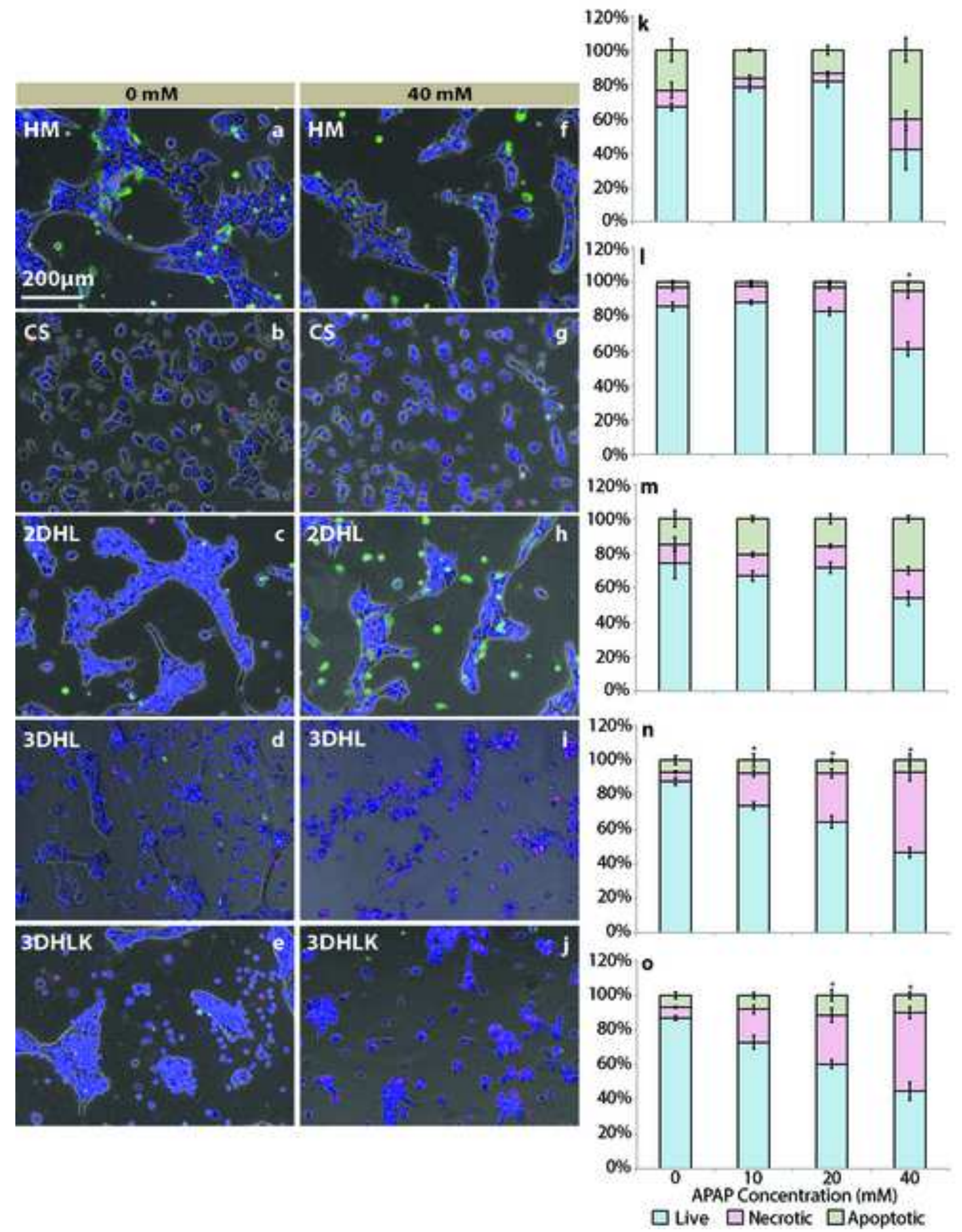




\section{Figure 4}

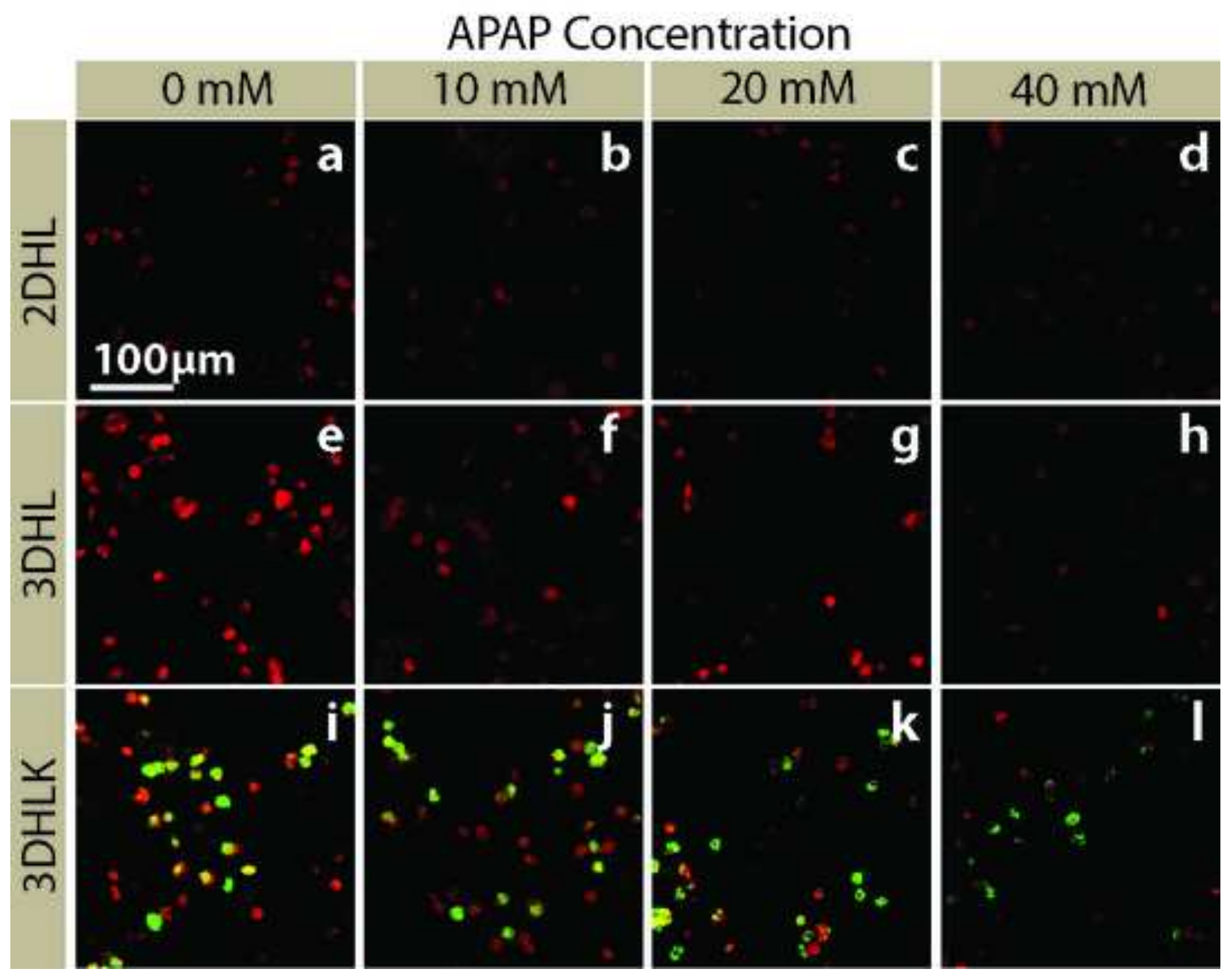




\section{Figure 5}

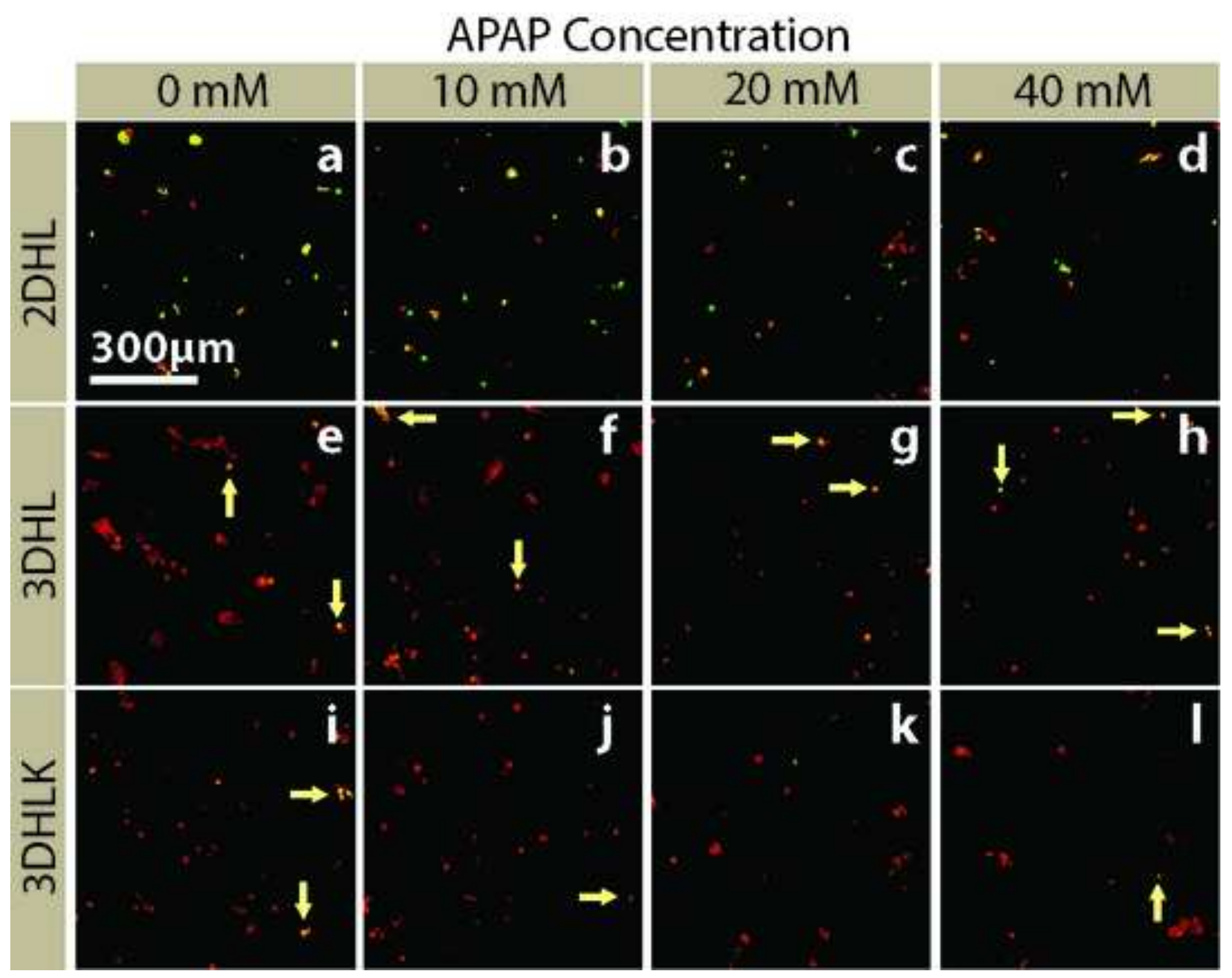


Figure 6

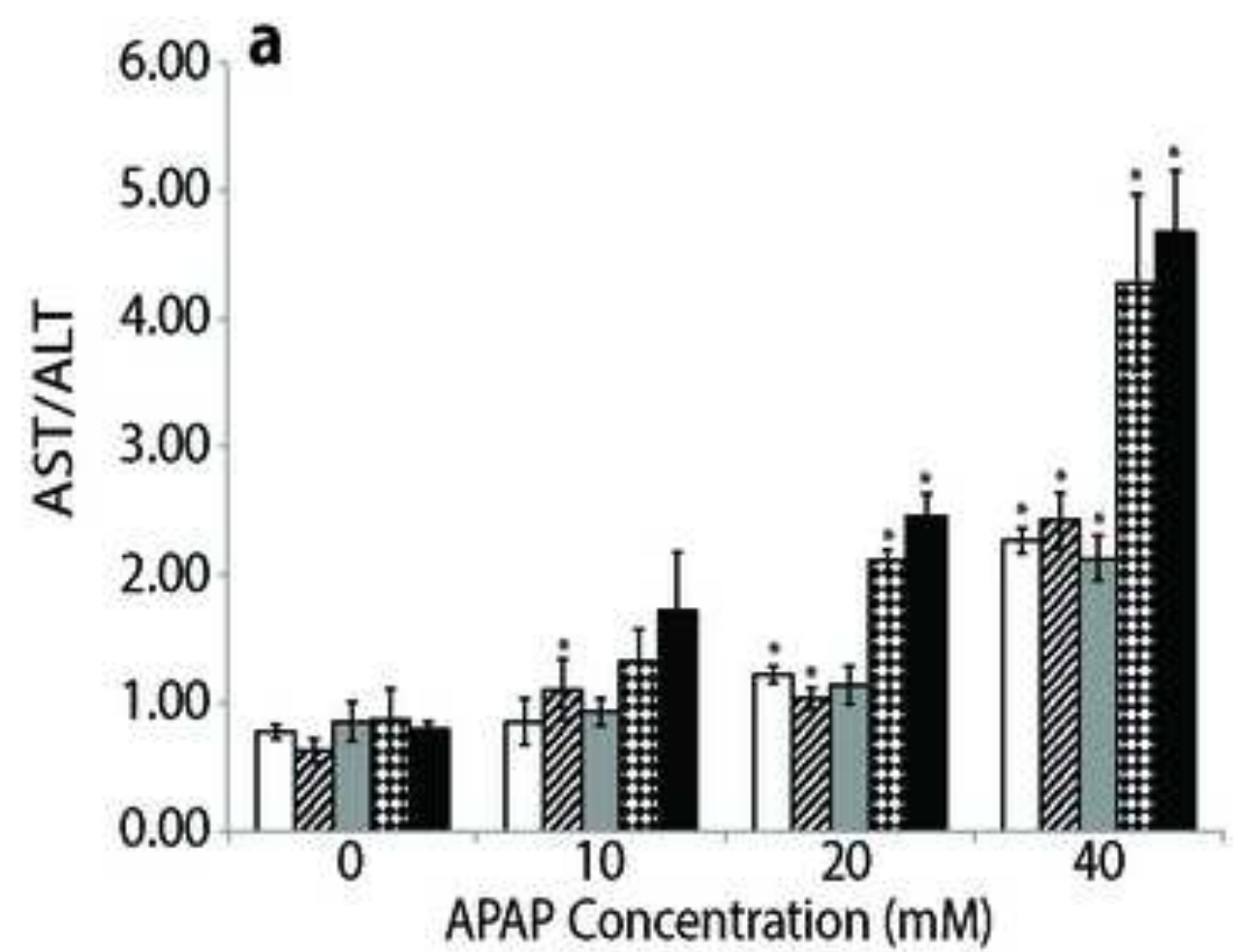

$\square$ Q $\square$ 2DHL @3DHL $\square$ 3DHLK

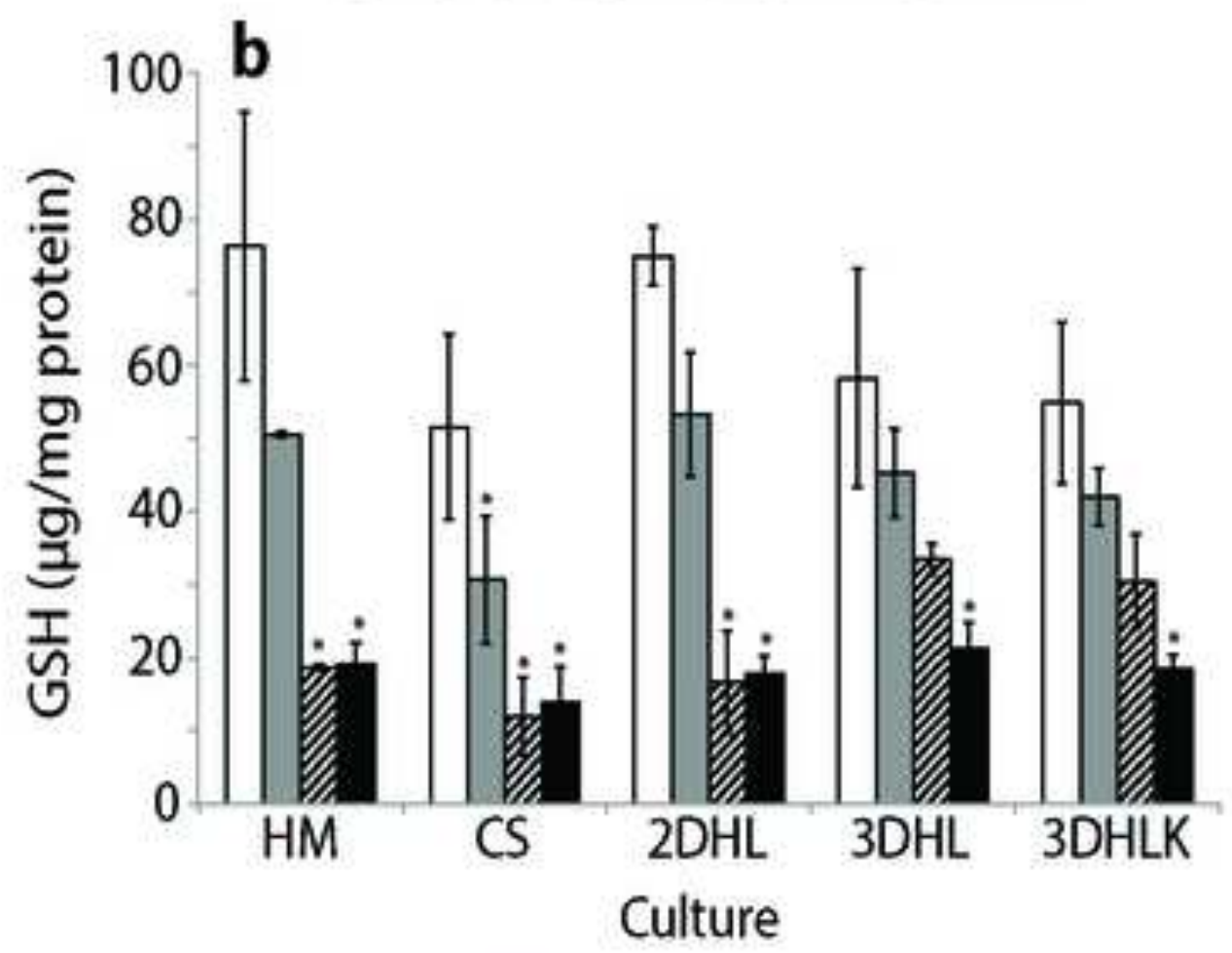

$\square 0 \mathrm{mM} \square 10 \mathrm{mM} \square 20 \mathrm{mM} \square 40 \mathrm{mM}$ 
Figure 7
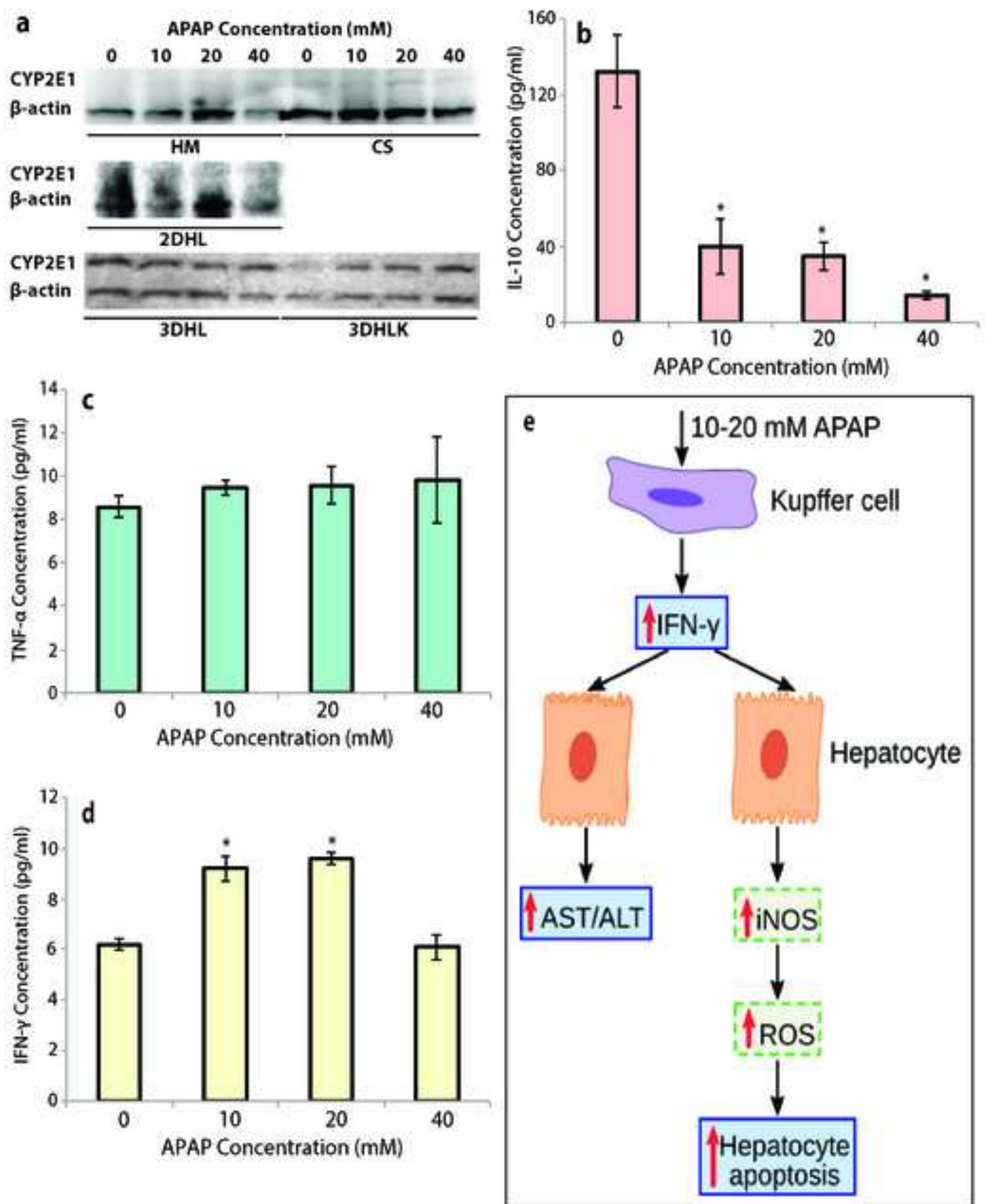